\title{
Leukocytes mediate disease pathogenesis in the Ndufs4(KO) mouse model of Leigh syndrome
}

\author{
Julia C. Stokes, ${ }^{1}$ Rebecca L. Bornstein,, ${ }^{1,2}$ Katerina James, ${ }^{1}$ Kyung Yeon Park, ${ }^{1}$ Kira A. Spencer, ${ }^{1}$ \\ Katie Vo, ${ }^{1}$ John C. Snell, ${ }^{1}$ Brittany M. Johnson, ${ }^{3}$ Philip C. Morgan,, ${ }^{1,4}$ Margaret M. Sedensky, ${ }^{1,4}$ \\ Nathan A. Baertsch, ${ }^{1,5}$ and Simon C. Johnson ${ }^{1,2,3,4}$ \\ 'Center for Integrative Brain Research, Seattle Children's Research Institute, Seattle, Washington, USA. ${ }^{2}$ Department of \\ Laboratory Medicine and Pathology, ${ }^{3}$ Department of Neurology, ${ }^{4}$ Department of Anesthesiology and Pain Medicine, and \\ ${ }^{5}$ Department of Pediatrics, University of Washington, Seattle, Washington, USA.
}

Symmetric, progressive, necrotizing lesions in the brainstem are a defining feature of Leigh syndrome (LS). A mechanistic understanding of the pathogenesis of these lesions has been elusive. Here, we report that leukocyte proliferation is causally involved in the pathogenesis of LS. Depleting leukocytes with a colony-stimulating factor 1 receptor inhibitor disrupted disease progression, including suppression of CNS lesion formation and a substantial extension of survival. Leukocyte depletion rescued diverse symptoms, including seizures, respiratory center function, hyperlactemia, and neurologic sequelae. These data reveal a mechanistic explanation for the beneficial effects of mTOR inhibition. More importantly, these findings dramatically alter our understanding of the pathogenesis of LS, demonstrating that immune involvement is causal in disease. This work has important implications for the mechanisms of mitochondrial disease and may lead to novel therapeutic strategies.

Authorship note: JC Stokes, RLB, and $\mathrm{KJ}$ contributed equally to this work.

Conflict of interest: The authors have declared that no conflict of interest exists.

Copyright: (c) 2022, Stokes et al. This is an open access article published under the terms of the Creative Commons Attribution 4.0 International License.

Submitted: November 8, 2021

Accepted: January 19, 2022

Published: March 8, 2022

Reference information: /CI Insight. 2022;7(5):e156522.

https://doi.org/10.1172/jici.

insight.156522.

\section{Introduction}

Grouped, genetic mitochondrial diseases (GMDs) are the most common cause of heritable metabolic disease and among the most common causes of pediatric neurologic dysfunction $(1,2)$. GMDs are genetically and clinically heterogeneous, grouping into clinically defined syndromes (2). Subacute necrotizing encephalopathy, or Leigh syndrome (LS), is the most common form of pediatric GMD. LS presents as a multisystem disorder with metabolic, neurologic, and musculoskeletal symptoms (3). Patients with LS are often born healthy, with symptoms presenting in the first years of life. Symmetric, progressive, necrotizing brainstem lesions are a defining feature of LS. A mechanistic understanding of these lesions has been elusive, and no effective clinical interventions exist. Inhibition of the mechanistic target of rapamycin (mTOR) attenuates disease in the $N d u f s 4(\mathrm{KO})$ mouse model of LS $(4,5)$, and mTOR inhibitors appears to benefit some patients $(6,7)$. The precise mechanism(s) of these benefits have been a mystery.

Following the 2013 report that mTOR inhibition significantly attenuates disease in the $N d u f_{s} 4(\mathrm{KO})$ model, great effort has been directed at defining the mechanistic underpinning of this disease attenuation. Subsequent studies have implicated multiple putative pathways, including metabolism, neurotransmitter abundance, PKC activity, redox, and transcription (5, 6, 8-11). However, no single downstream pathway has been found to reproduce the benefits of mTOR inhibition, suggesting the role of mTOR may not be defined by one discrete downstream process.

The studies detailed in this report had the goal of assessing whether the role of mTOR in the $N d u f s 4(\mathrm{KO})$ model is defined by upstream signaling, specifically, whether signaling through PI3K contributes to LS and PI3K suppression attenuates disease (for more detail, see Supplemental Figure 1; supplemental material available online with this article; https://doi.org/10.1172/jci.insight.156522DS1). Here, we report that inhibition of the PI3K catalytic subunit $\gamma$ isoform $\mathrm{p} 110 \gamma / \mathrm{PI} 3 \mathrm{~K} \gamma$, expressed primarily in leukocytes, recapitulates the benefits of mTOR inhibition, while inhibition of other PI3K isoforms does not (12). We show that directly targeting leukocyte proliferation via colony-stimulating factor 1 receptor (CSF1R) inhibition dramatically attenuates disease. CSF1R inhibition blocks CNS lesion formation, prevents neurologic signs, 
and rescues disease sequelae including hyperlactemia, seizures, hypoglycemia, and anesthetic responses, as well as significantly extending survival.

Note: while Iba1 ${ }^{+}$(ionized calcium-binding adapter molecule 1, aka allograft inflammatory factor 1 , Aif1) cells are referred to as microglia throughout LS literature, we avoid this language for reasons detailed in the discussion section.

\section{Results}

Isoform-specific pharmacologic targeting of PI3K $\gamma$ significantly attenuates disease in the Ndufs $4(K O)$ mouse. To determine whether the benefits of mTOR inhibition in $N d u f s(\mathrm{KO})$ mouse result from disruption of PI3K-mediated signaling, we treated animals from weaning (postnatal day 21, P21) with potent, orally available, isoform-specific inhibitors of the catalytic subunits of PI3K: BYL719, GSK2636771, CAL-101, and IPI-549 - inhibitors of $\mathrm{p} 110 \alpha, \mathrm{p} 110 \beta, \mathrm{p} 110 \delta$, and $\mathrm{p} 110 \gamma$, respectively

Control-treated $N d u f s 4(\mathrm{KO})$ animals displayed normal health early in life but rapidly developed progressive neurologic symptoms starting around P37, and death occurred by approximately P80 (Figure 1A). After around P37, animals developed progressive, degenerative lesions in the brainstem and cerebellum. Signs of neurologic decline include forelimb clasping, ataxia, and circling. Animals developed cachexia without anorexia (Supplemental Figure 2); this is the most frequent proximal cause of death, as euthanasia due to loss of body mass is the approved endpoint typically reached first (death by disease is generally not allowed in IACUC-approved animal work). The mechanistic underpinnings of this cachexia are unknown.

Treatment with the $\mathrm{p} 110 \alpha, \mathrm{p} 110 \beta$, and $\mathrm{p} 110 \delta$ inhibitors provided only modest $(\sim 10 \%$ or less) benefits to survival (Figure 1, B and C). In contrast, treatment with the p110 $\gamma$ inhibitor IPI-549 increased survival similarly to mTOR inhibition - median survival in IPI-549-treated $N d u f s 4(\mathrm{KO})$ mice was approximately 110 days versus approximately 110 and approximately 60 for rapamycin-treated and untreated animals, respectively. Drug dosing was based on published studies (13-16) (see Supplemental Methods and Discussion). Treatments led to similar tissue levels (Supplemental Figure 3).

Each of the PI3K catalytic subunit inhibitors modestly delayed at least some overt signs of neurologic disease (clasping, circling, ataxia), but IPI-549 provided the greatest benefits (Figure 1, D-G). In contrast, only IPI-549 delayed/prevented cachexia (Figure 1, G-I), and only IPI-549 improved Ndufs4(KO) performance on a rotarod assay, which assesses neurologic function and overall health (Figure 1J). Further, IPI-549 alone prevented progressive hypoglycemia (Figure $1 \mathrm{~K}$ ). In addition to being the only PI3K catalytic subunit inhibitor to increase survival (detailed above), IPI-549 alone qualitatively affected cause of death (Figure 1L).

mTOR inhibition with ABI-009 (nab-rapamycin, a rapamycin formulation for injection, see Supplemental Methods) at doses sufficient to attenuate disease significantly reduced developmental weight gain and maximum body size (Figure 1, I, M, and N), as we have reported previously for oral and injected rapamycin $(4,5)$. IPI-549 and mTOR inhibition similarly modified disease, while IPI-549 had a milder impact on growth (Figure 1M). IPI-549 also did not cause low glucose as we have observed with chronic rapamycin treatment (see ref. 17). In contrast, BYL719 severely impaired size, significantly more than IPI-549, while not rescuing disease (Figure 1, B, H, I, M, and N); these data indicate mTOR inhibition does not benefit LS through actions on insulin/insulin like growth factor 1 signaling, which is mediated by $\mathrm{p} 110 \alpha(18)$.

We also tested a pan-PI3K inhibitor, BKM-120, finding that while well tolerated in adult mice at 60 $\mathrm{mg} / \mathrm{kg} / \mathrm{d}$ (19), BKM-120 was not tolerated at 50 or $100 \mathrm{mg} / \mathrm{kg} / \mathrm{d}$ when administered from weaning (Supplemental Figure 4). Given our IPI-549 results, we did not explore this strategy further.

mTOR inhibition reduces Iba1 leukocyte proliferation in vitro. $\mathrm{p} 110 \gamma$ is primarily expressed in leukocytes (Supplemental Figure 5), including the brain's resident macrophages, microglia. Lesions in LS are characterized in part by the accumulation of $\mathrm{Iba}^{+}$cells (typically referred to as "microgliosis"; see Discussion regarding use of leukocyte versus microglia). This has widely been thought a secondary reaction to CNS cell death caused by some combination of "energetic depletion," ROS damage, lactic acidosis, and excitotoxicity $(3,20)$. Given that $\mathrm{p} 110 \gamma$ and mTOR inhibitors provide similar benefits, we reasoned that leukocyte (including microglia) proliferation may be causal in CNS lesion formation and degeneration, rather than simply a secondary response.

To assess whether mTOR inhibition affects leukocyte/microglia proliferation in vitro, we tested the impact of ABI-009 (aka nab-rapamycin, see refs. 17, 21), a water-soluble nanoparticle formulation of rapamycin (see Supplemental Methods), on Iba ${ }^{+}$cells in a mixed brain cell culture assay, comparing the impact with that of 
A

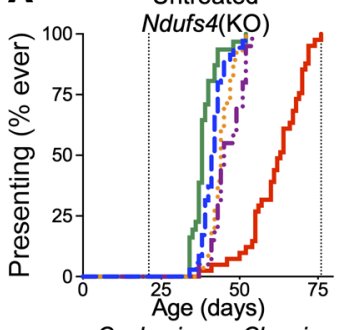

- Cachexia - Clasping Ataxia - Circling - Death

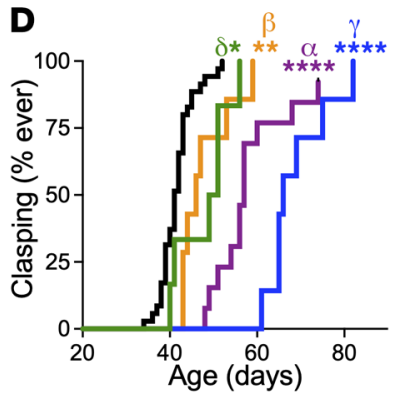

H

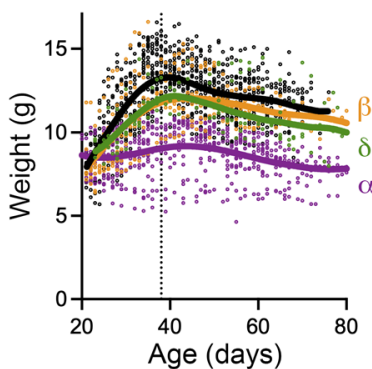

B

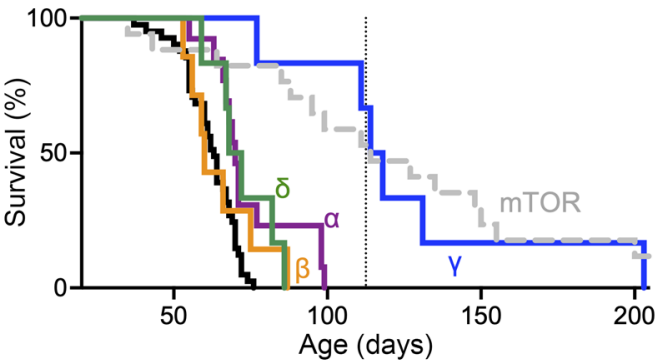

C

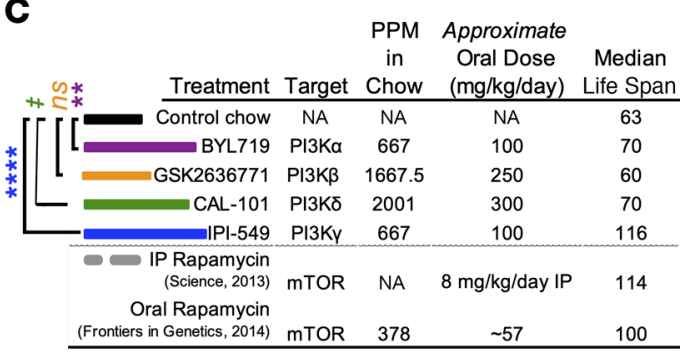

E

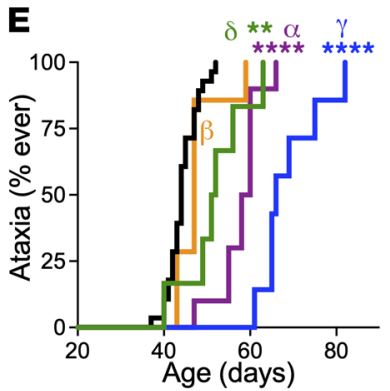

I

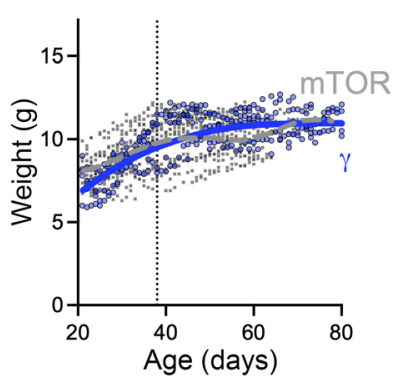

$\mathbf{F}$

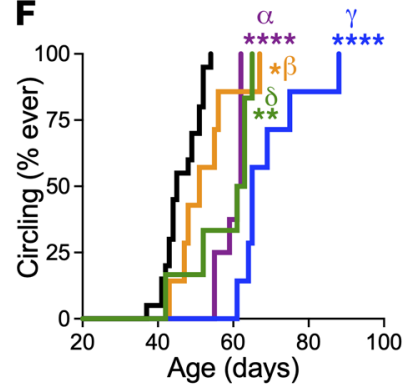

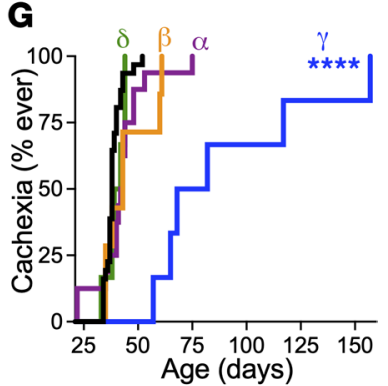

$\mathbf{J}$
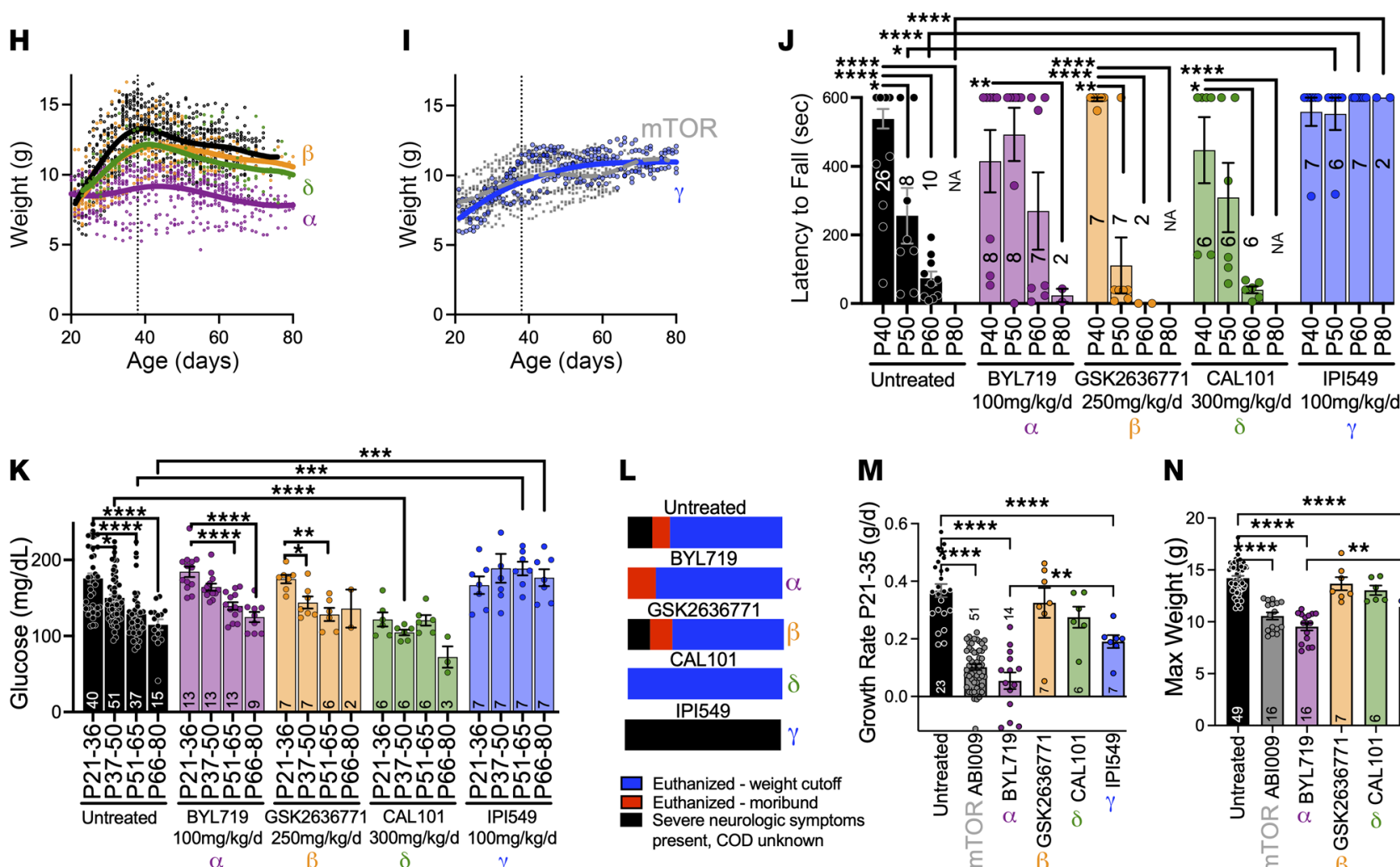

ᄂ

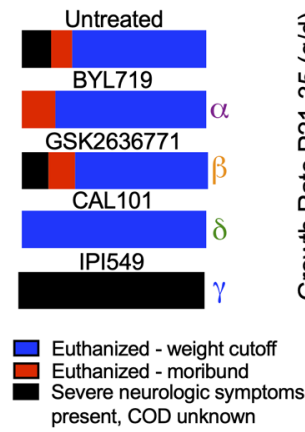

M

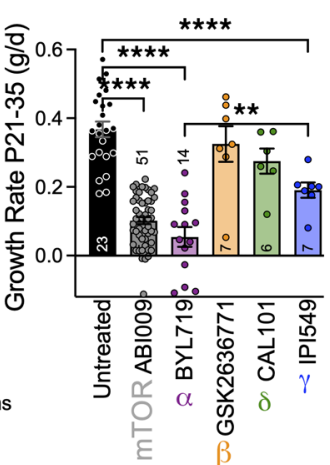

$\mathbf{N}$

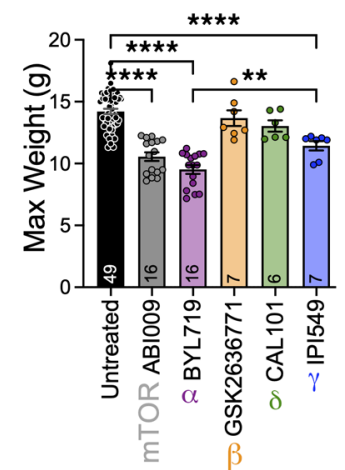

Figure 1. Isoform-specific inhibition of PI3K catalytic subunit $\mathrm{p} 110 \gamma$, but not $\mathrm{p} 110 \alpha, \mathrm{p} 110 \beta$, or p110 $\delta$, significantly attenuates disease in the Ndufs4(KO) mouse model of LS. (A) Age of cachexia $(n=31)$, clasping $(n=35)$, ataxia $(n=28)$, and circling $(n=20)$ onset and of death $(n=41)$ (see Methods for symptoms, replicates, scoring). (B and C) Survival (B) and life span and dosage data (C) for Ndufs4(KO) mice administered control chow

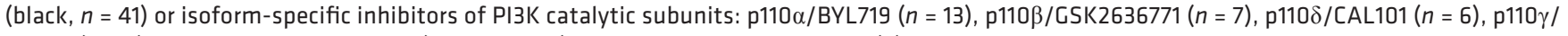
IPI549 $(n=6)$. Rapamycin for reference (see refs. 4, 5); line, median for rapamycin. (C) Color key for $\mathbf{B}$ and $\mathbf{D}-\mathbf{C}$, dosing, and median life spans. ${ }^{\ddagger} P=$ 0.017, ${ }^{* *} P<0.005$, and ${ }^{* * *} P<0.0001$, log-rank test (Bonferroni-corrected significance threshold, BCST: $\left.P<0.015\right)$. (D-G) Clasping (D), ataxia (E), circling (F), and cachexia (G) in Ndufs4(KO) mice treated with BYL719 $(n=12,10,8,16)$, GSK2636771 $(n=7,7,7,7)$, CAL101 $(n=6,6,6,6)$, or IPI549 ( $n=7$, $7,7,6)$. Treatment indicated by color (see $\mathbf{C}$ ) and $\mathrm{p} 110 \alpha / \beta / \delta / \gamma$ symbols. Control-treated $n$ s as in $\mathbf{A} .{ }^{*} P<0.015,{ }^{*} P<0.005$, ${ }^{* *} P<0.0005$, and ${ }^{* * * *} P$ $<0.0001$ by log-rank test vs. untreated Ndufs4(KO) (BCST: $P<0.015$ ). (H and I) Ndufs4(KO) weight by age and treatment (indicated by color and symbol; mTOR, ABI009 treatment); local regression (Lowess) curve overlaid. ns as in B. (J) Performance of Ndufs4(KO) mice on a rotarod assay. (K) Blood glucose by age (see Methods). (J and K) $n$ provided as numbers within/above bars. ${ }^{*} P<0.015,{ }^{* *} P<0.005,{ }^{* * *} P<0.0005$, and ${ }^{* * * *} P<0.0001$ by Welch's $t$ test (treated vs. untreated BCST: $P<0.015$; vs. baseline within same treatment BCST: $P<0.0167$ ). For rotarod, when animals die before 
P80, $t$ tests are interpreted as significant. (L) Cause of death in survival studies. $n$ values as in $\mathbf{A}$ and $\mathbf{B}$. (M and $\mathbf{N}$ ) Growth rate P21-P35 (M) and maximum weight during life (N). ns indicated within bars. ${ }^{*} P<0.0033$, ${ }^{* *} P<0.0005$, and ${ }^{* * *} P<0.0001$, unpaired, unequal variances (Welch's) $t$ test (BCST: $P<0.0033$ ). Data represent mean, error bars \pm SEM, unless otherwise stated.

pexidartinib/PLX3397, a CSF1R inhibitor that blocks leukocyte survival signaling (22) (Figure 2A). ABI-009 and pexidartinib both reduced the fraction of $\mathrm{Iba}^{+}$cells in mixed neonatal brain cell cultures in a dose-dependent manner. The maximum effects of ABI-009 and pexidartinib were about $50 \%$ and about $100 \%$ depletions, respectively. Accordingly, mTOR inhibition does appear to preferentially limit leukocyte proliferation compared with other cell types, but the potency is limited compared with directly targeting leukocyte survival.

Leukocyte depletion prevents CNS lesions and neurologic sequelae. Taken together, our data suggested that leukocyte proliferation may causally drive disease in LS.

To test this model, we treated $N d u f f(\mathrm{KO})$ and control animals with 100,200 , or $300 \mathrm{mg} / \mathrm{kg} / \mathrm{d}$ pexidartinib in normal mouse chow (dosing approximated based on food consumption, see Supplemental Methods; tissue drug levels in Supplemental Figure 6; note - higher doses led to a change in mouse coat color, consistent with reports of hair whitening in humans; Figure 2).

Treatment with $300 \mathrm{mg} / \mathrm{kg} / \mathrm{d}$ pexidartinib led to a complete (by IHC) prevention of brainstem and cerebellar (Figure 2) lesions in $N d u f s 4(\mathrm{KO})$ mice, even at ages far beyond the maximum survival of untreated animals. Critically, both accumulation of $\mathrm{Iba}^{+}{ }^{+}$cells and astrocytosis were completely prevented by pexidartinib, indicating that astrocyte involvement is secondary to leukocyte activity.

Consistent with these findings, pexidartinib dose dependently delayed the onset and reduced the incidence of behavioral signs of neurodegeneration - forelimb clasping, ataxia, and circling (Figure 2, G-I). Pexidartinib treatment also rescued performance on the rotarod assay (Figure 2J).

Impaired respiration, which is a brainstem function, is a proximal cause of death in patients with LS and has been reported to be a proximal cause of death in $N d u f 4(\mathrm{KO})$ mice not euthanized (23). We performed plethysmography in untreated and $300 \mathrm{mg} / \mathrm{kg} / \mathrm{d}$ pexidartinib-treated mice to assess respiratory function (see ref. 24 for diagram and Supplemental Methods for details). Severe defects in respiratory function were present in untreated $N d u f 4(\mathrm{KO})$ mice at about P70 in respiratory frequency, incidence of amplitude and frequency irregularities, and responses to increased $\mathrm{CO}_{2}$ (Figure 2). Treatment with $300 \mathrm{mg} / \mathrm{kg} / \mathrm{d}$ pexidartinib completely prevented each of these respiratory defects (Figure 2).

Leukocyte depletion rescues neuroinflammation outside of lesions. Overt lesions in the brainstem and cerebellum underlie many features of LS, while inflammation in other CNS regions has not been carefully studied. Given the robust impact of pexidartinib in preventing lesions, we wondered whether neuroinflammation is present, and responsive to pexidartinib, in other brain regions. To assess this possibility, we analyzed Iba $1^{+}$ and $\mathrm{GFAP}^{+}$(astrocyte) cell numbers in cortex and in brainstem tissue outside overt lesions. Untreated Ndufs4(KO) mice showed increases in Iba1 ${ }^{+}$cells and astrocytes in nonlesion CNS tissue, while $300 \mathrm{mg} /$ $\mathrm{kg} / \mathrm{d}$ pexidartinib prevented both markers of neuroinflammation (Figure 3, A-E; see also Supplemental Figure 7). Notably, pexidartinib treatment led to a near-complete depletion of $\mathrm{Iba}^{+}{ }^{+}$cells while astrocytes were rescued to control levels, again indicating that astrocytosis is secondary to leukocyte involvement. As with lesions, the benefits of pexidartinib extended to ages far beyond the maximum life span of untreated $N d u f 4(\mathrm{KO})$ mice (see Figure 3 legend for details).

Pexidartinib treatment prevents rotarod-induced seizures. Given the inflammation in multiple CNS regions, we next wondered if LS features not previously linked to the lesions, such as seizures, might also be rescued by targeting leukocytes. Epileptic seizures are common in LS and often refractory to standard therapies (25).

The rotarod assay provided a mild epileptogenic stimulus in the $N d u f s(\mathrm{KO})$ model: seizures occurred in approximately $30 \%$ of untreated Ndufs $4(\mathrm{KO})$ mice, but were not observed in control animals, during rotarod at age P30 (17) (Figure 3F and Supplemental Methods). We performed rotorod assay on control and pexidartinib-treated Ndufs $4(\mathrm{KO})$ mice to assess whether targeting leukocytes impacts seizures. Incidence and time to seizure were both significantly reduced by pexidartinib (Figure 3, F and G).

Hyperlactemia is prevented by rapamycin, IPI-549, and pexidartinib. Metabolic defects are major sequelae of GMDs. As noted, treatment with pexidartinib prevented both cachexia and hypoglycemia in the $N d u f 4(\mathrm{KO})$ mice in a dose-dependent manner (Figure 3, H-J) (5), consistent with leukocytes playing a role in metabolic derangements. We next considered the possibility that leukocyte activity may drive other metabolic sequelae in LS. 
A

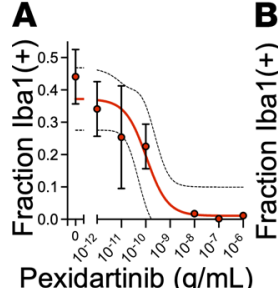

Pexidartinib $(\mathrm{g} / \mathrm{mL})$
B

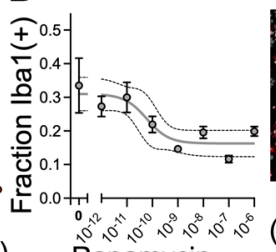

Rapamycin (ABI-009) $(\mathrm{g} / \mathrm{mL})$
C $\quad$ IBA1 (red) DAPI (gray)

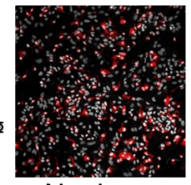

$\begin{array}{cc}\text { No drug } & 10 \mathrm{ng} / \mathrm{ml} \\ \text { (DMSO only) } & \text { Pexidartinib }\end{array}$

Pexidartinib

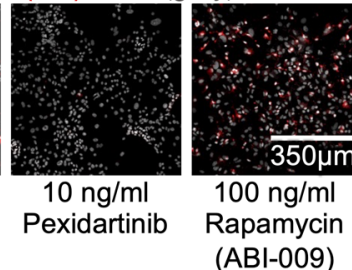

(ABI-009)

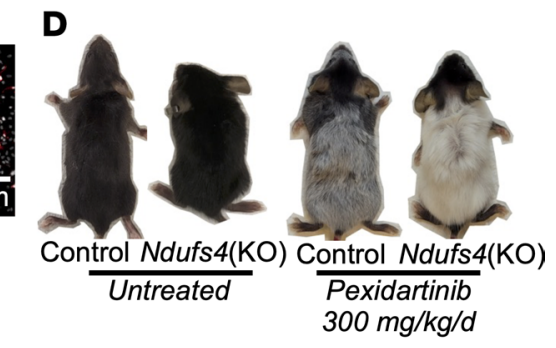

IBA1(red) GFAP(green) DAPI(blue)

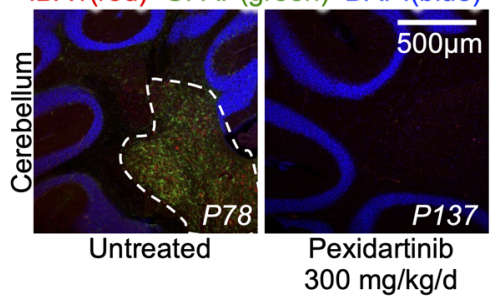

E

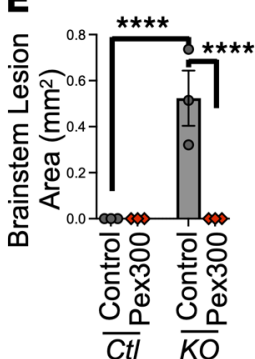

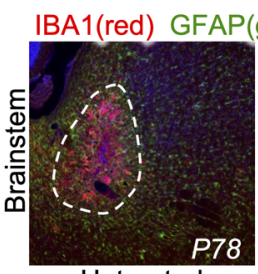

Untreated
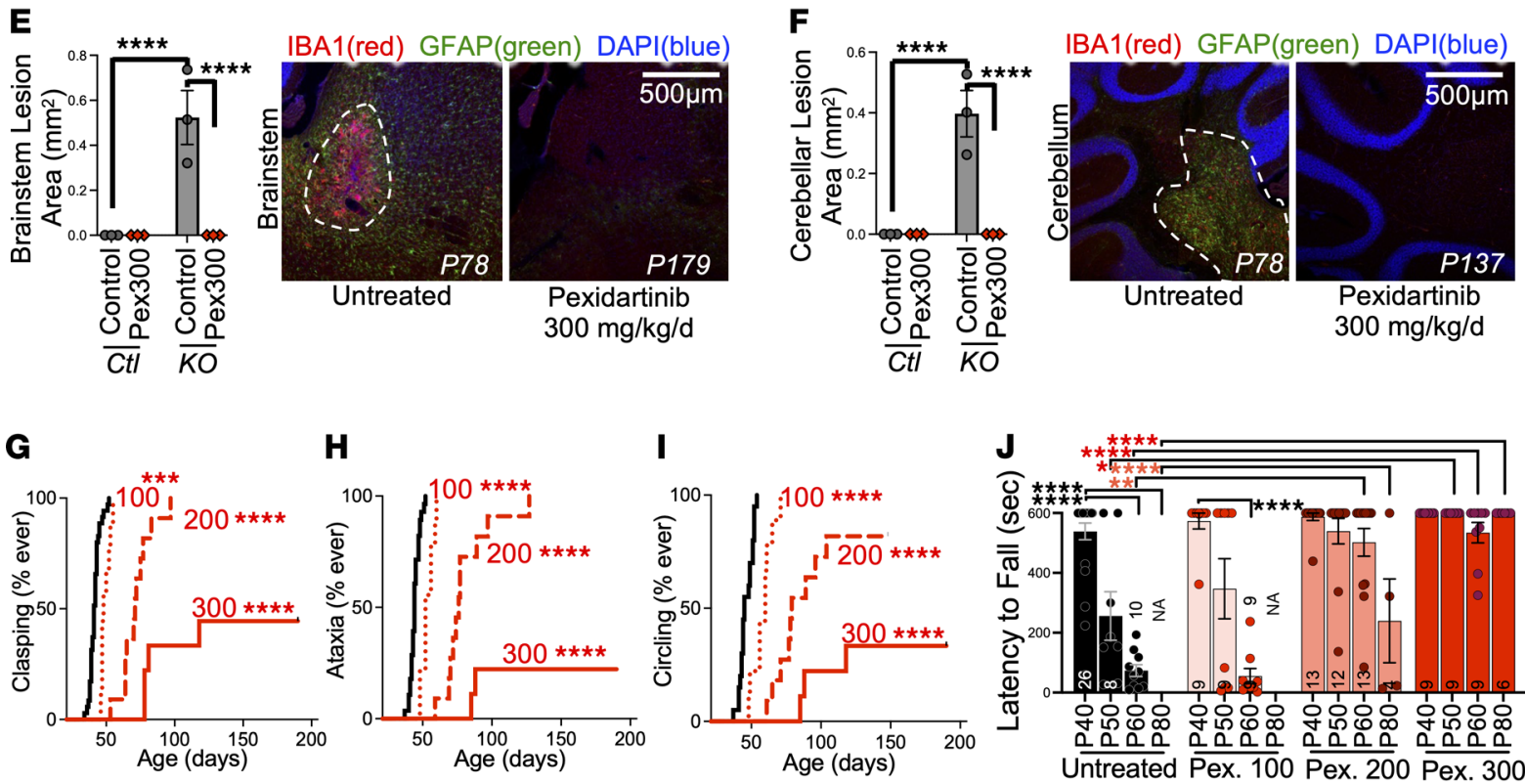

$\mathbf{K}$

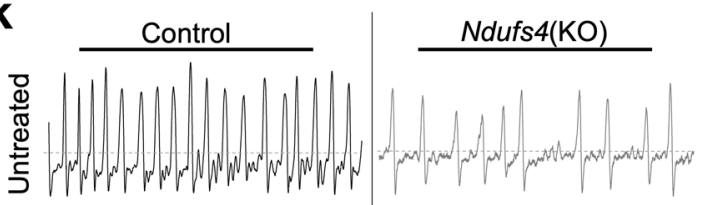

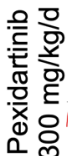
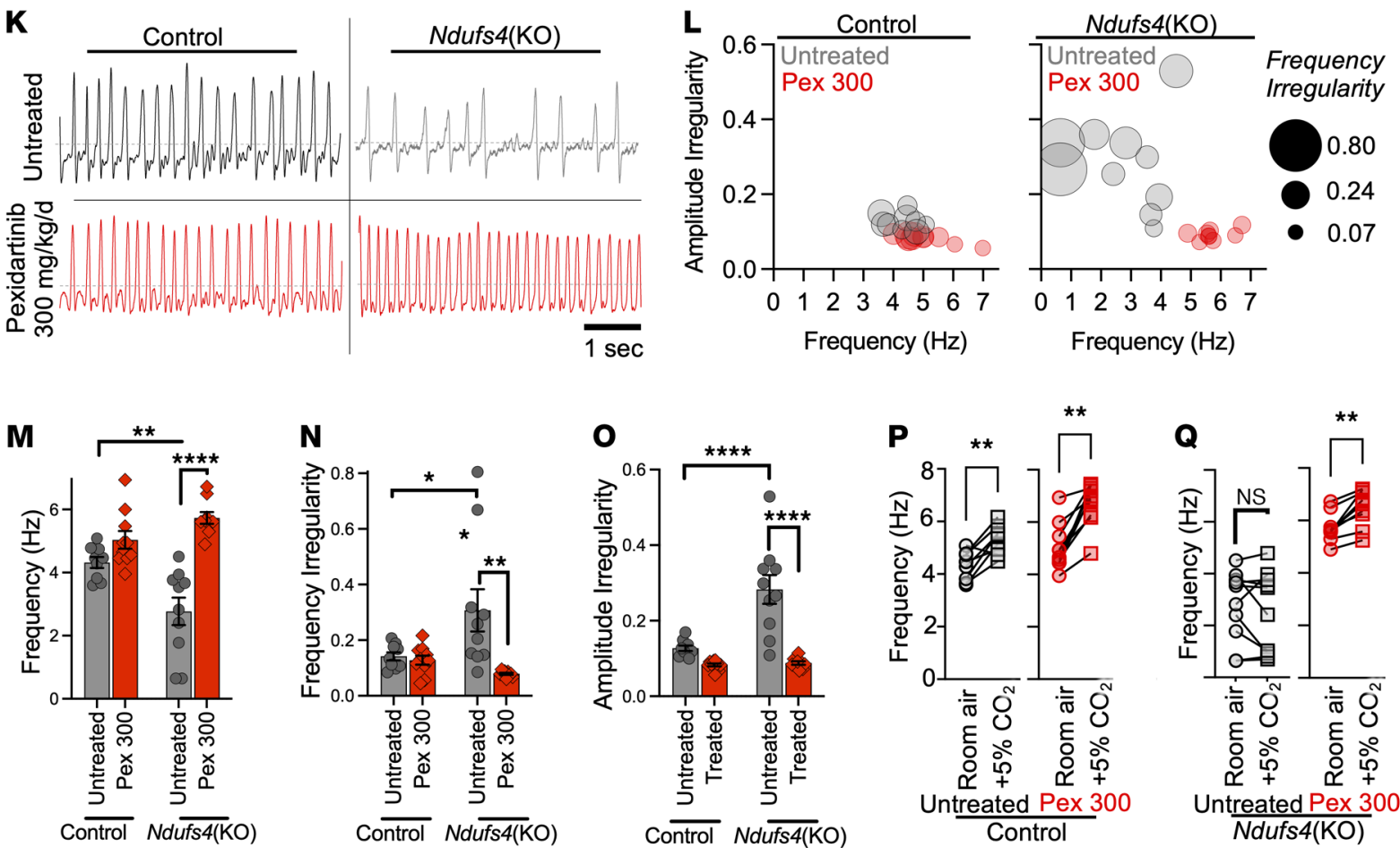

Figure 2. Leukocyte depletion prevents CNS lesions and significantly attenuates disease in the Ndufs4(KO) model of LS. (A and B) Dose-dependent impact of the CSF1R inhibitor pexidartinib (A) and rapamycin (ABI-009 formulation) (B) on the fraction of Iba1+ leukocytes (likely microglia given cell origins, see Methods) in mixed primary brain cultures. Error bars show \pm SEM. Dashed lines, $95 \%$ confidence interval for inhibitor vs. response (3 parameters) least squares fit. $n=3$ replicates/concentration for rapamycin, $6 /$ condition for pexidartinib (data representative of 3 independent experiments). 
(C) Representative images of mixed primary brain cultures from $\mathbf{A}$ and $\mathbf{B}$ stained with an anti-lba1 antibody (red) and DAPI (blue, nuclei). Scale bar in rapamycin image is representative for all. (D) Representative pictures of control and Ndufs4(KO) animals fed control diet or $\sim 300 \mathrm{mg} / \mathrm{kg} / \mathrm{d} \mathrm{pexidartinib}$ chow. (E and $\mathbf{F})$ Brainstem (E) and cerebellar peduncle (F) lesion size (area of lesion in central slice in serial sectioning, see Methods) in control-treated (Untreated) and $300 \mathrm{mg} / \mathrm{kg} / \mathrm{d}$ pexidartinib (Pex300) treated control (CtI) and Ndufs4(KO) (KO) animals. Quantification and representative images provided for each region. Representative images are only provided for Ndufs4(KO) animals as control mice do not develop lesions. See Figure 3 for quantification of Iba1+ leukocytes and GFAP+ astrocytes in control and Ndufs4(KO) mice. Anti-lba1 antibody staining in red, DAPI (nuclei) in grayscale (see Methods). Lesion areas indicated by dashed white lines. Ages are noted in representative images (P78, etc.). ${ }^{* * *} P<0.0001$ by unpaired, unequal variances (Welch's) $t$ test. $n=3$ animals per condition. (G-I) Onset of clasping (G), ataxia (H), and circling (I) in Ndufs4(KO) mice fed control diet (black lines, $n$ as in Figure 1; see Methods) or administered pexidartinib at $100 \mathrm{mg} / \mathrm{kg} / \mathrm{d}$ (dotted red lines, $n=9,9,9), 200 \mathrm{mg} / \mathrm{kg} / \mathrm{d}$ (dashed red lines, $n=11,11,11$ ), or $300 \mathrm{mg} / \mathrm{kg} / \mathrm{d}$ (solid red lines, $n=9,9,9$ ). ${ }^{* *} P<0.0005$, and ${ }^{* * *} P<0.0001$ by log-rank test vs. untreated Ndufs4(KO) animals (Bonferroni significance threshold $=P<0.0167$ ). (J) Performance of control- and pexidartinib-treated animals on a rotarod assay. ${ }^{* *} P<0.005$, and ${ }^{* * * *} P<0.0001$ by unpaired, unequal variances (Welch's) $t$ test (BCST $={ }^{*} P<0.0167$ for both comparisons between pexidartinib-treated and control untreated mice and for comparisons to baseline within same treatment). Replicates (ns) are shown by vertically oriented numbers within/above bars. For rotarod, where animals do not survive to P80, $t$ test undefined but interpreted as highly significant (see Methods). (K) Representative traces of respiratory (breathing) activity in P70 ( \pm 2 days) control and Ndufs $4(\mathrm{KO})$ mice control treated or fed $300 \mathrm{mg} / \mathrm{kg} / \mathrm{d}$ pexidartinib chow. (L) Multivariable plotting of respiratory amplitude irregularity, frequency, and frequency irregularity in P7O ( \pm 2 days) control and Ndufs $4(\mathrm{KO})$ mice fed control chow or administered $300 \mathrm{mg} / \mathrm{kg} / \mathrm{d} \mathrm{pexidartinib.}$ (M) Frequency of breathing (representative data in $\mathbf{K}$ ). ( $\mathbf{N}$ and $\mathbf{0})$ Single-variable analysis of data in $\mathbf{L}$. (M-O) Data points represent individual animals, ${ }^{*} P<0.05$, ${ }^{* *} P<0.005$, and ${ }^{* * *} P<0.0005$ by 2 -way ANOVA with Tukey's multiple-testing correction-adjusted $P$ values for pairwise comparisons. Each comparison is pairwise. ( $\mathbf{P}$ and $\mathbf{Q})$ Respiratory responses to increased environmental $\mathrm{CO}_{2}$. Pairwise data shown for responses in individual mice.

${ }^{* *} P<0.005$ by Wilcoxon matched pairs signed-rank test. For all panels, data represent mean, error bars $\pm \mathrm{SEM}$, unless otherwise stated. (M-Q) $n=9$ for control animal data sets, 10 for Ndufs4(KO) data sets.

Abnormally high blood or CNS lactate (often assessed by lactate/pyruvate ratio) is frequently reported in LS and some other forms of GMD (26-29). In addition, increased lactate is a feature of LS CNS lesions when imaged by magnetic resonance spectroscopy (MRS), and increased intracerebral lactate by MRS has been reported in $N d u f s 4(\mathrm{KO})$ mice (30-32). The cellular origins of increased lactate in GMD have not been defined. Leukocytes are highly glycolytic (33), so a role for leukocytes in contributing to lactate appeared reasonable.

To probe hyperlactemia in untreated $N d u f s 4(\mathrm{KO})$ mice, we measured blood lactate at baseline and in response to a glucose bolus in a glucose tolerance test (GTT) paradigm. Clearance of glucose was not significantly altered in the $N d u f s 4(\mathrm{KO})$ animals, and blood lactate was not increased in $N d u f s 4(\mathrm{KO})$ animals at baseline; however, exposure of $N d u f s(\mathrm{KO})$ animals to a glucose bolus resulted in a significant rise in blood lactate not observed in control mice (Figure 3, K and L, and Supplemental Figure 8). Notably, this glucose-induced hyperlactemia occurred only in $N d u f s 4(\mathrm{KO})$ mice older than P37, when CNS symptoms present. Pexidartinib treatment prevented the glucose-induced hyperlactemia, supporting the leukocyte model.

Given the clinical importance of hyperlactemia, we next tested the impact of mTOR or PI3K $\gamma$ inhibition on GTT-induced hyperlactemia, finding that both ABI-009 and IPI-549 prevented hyperlactemia in response to a glucose bolus (Figure 3, M-O). Only rapamycin/ABI-009 significantly reduced baseline lactate.

Pexidartinib attenuates anesthetic sensitivities in the $N d u f s 4(K O)$ model. Hypersensitivity to both sedation and toxicity from volatile anesthetics is seen in mitochondrial mutants from invertebrates to mammals, including $N d u f s 4(\mathrm{KO})$ mice and human patients with electron transport chain complex I (ETC CI) defects $(34,35)$. Mechanisms are poorly understood, but off-target effects include metabolic disruption; low-dose isoflurane exposure leads to a blood lactate spike in $N d u f s 4(\mathrm{KO})$ mice but not control animals (Figure 3P).

Given the impact of pexidartinib on lactate during the GTT, we tested whether pexidartinib affects isoflurane-induced hyperlactemia. Treatment fully suppressed this lactate spike in $N d u f s(\mathrm{KO})$ animals (Figure $3 \mathrm{P}$ ).

To test whether pexidartinib affects hypersensitivity to sedation, we measured the tail-clamp MAC (see ref. 36) of isoflurane in control and $300 \mathrm{mg} / \mathrm{kg} / \mathrm{d}$ pexidartinib-treated $N d u f s 4(\mathrm{KO})$ mice (see Supplemental Methods for details). We tested P30 animals to assess sensitivity in the absence of overt neurologic disease. Pexidartinib modestly but significantly attenuated hypersensitivity in the $N d u f s 4(\mathrm{KO})$ mice (Figure $3 \mathrm{Q}$ ).

Pexidartinib increases Ndufs4(KO) life span, while drug toxicity limits survival. Pexidartinib extended survival of $N d u f s 4(\mathrm{KO})$ animals in a dose-dependent manner (Figure 4, A and B). Critically, the survival curves of control and $N d u f s(\mathrm{KO})$ animals treated with $300 \mathrm{mg} / \mathrm{kg} / \mathrm{d}$ pexidartinib overlapped, indicating that the treatment, rather than the underlying disease, is limiting survival at this dose. Consistent with this notion, the majority of high-dose pexidartinib animals did not show overt signs of neurologic sequelae prior to death; the proximal cause of death was unclear for both $N d u f 44(\mathrm{KO})$ and control animals at this dose (Figure 4C; see Discussion).

Chronic pexidartinib is associated with risk of serious cholestatic or mixed liver injury in human patients; hepatic function is carefully monitored in patients taking this drug (37). We tested blood alanine aminotransferase (ALT) and aspartate aminotransferase (AST), established blood markers for pexidartinib-induced 
A

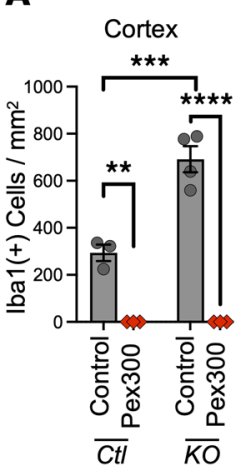

B

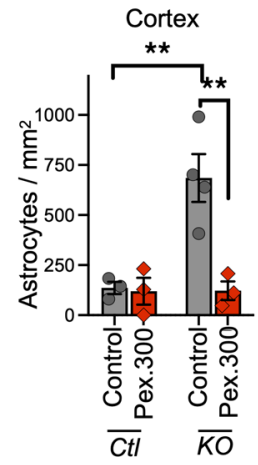

C

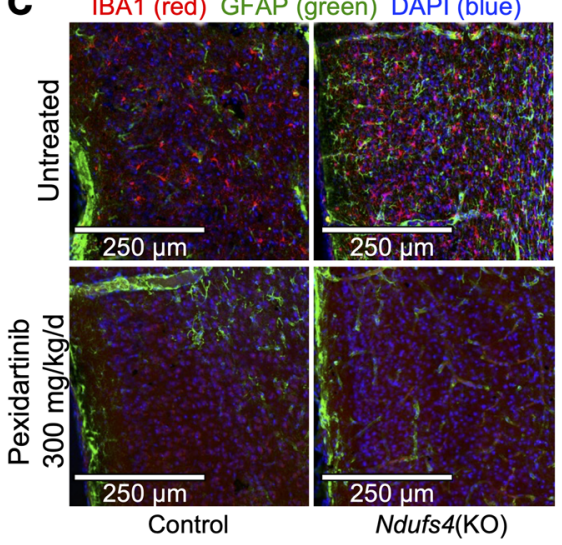

D Brainstem
(outside lesion)

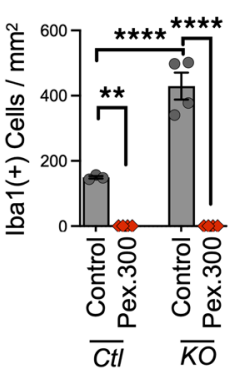

$\mathbf{E}$

Brainstem (outside lesion)

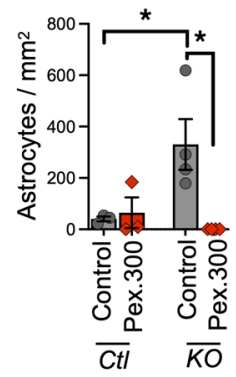

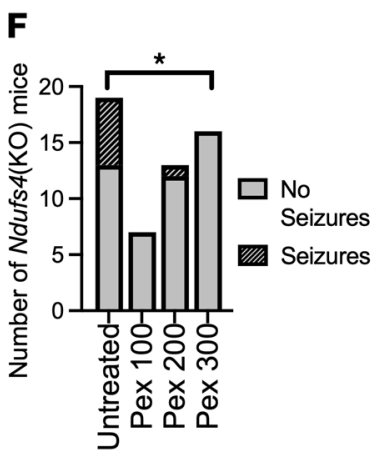
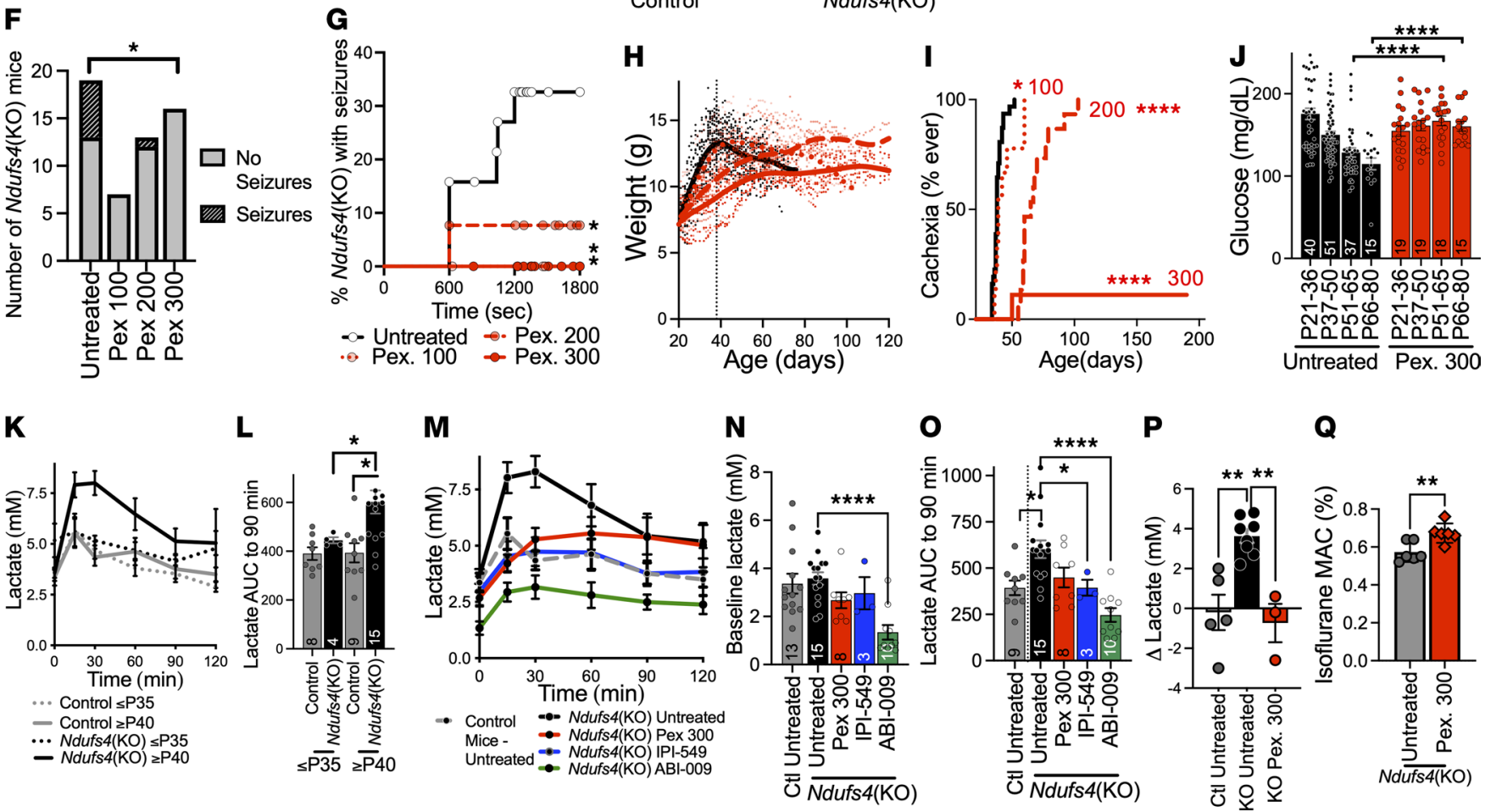

Figure 3. Leukocyte depletion prevents leukocyte/microglia accumulation and astrocytosis throughout the brain and rescues a range of systemic symptoms associated with LS in the Ndufs4(KO) mice. (A-C) Iba1+ leukocytes/microglia (A) (see Discussion) and GFAP astrocytes (B) in cortex of control- and pexidartinib-treated control and Ndufs4(KO) (see Methods). Data points represent individual animals. $n=4$ for untreated $N d u f s 4$ (KO), 3 for other groups. (C) Representative images of cortex. ( $\mathbf{D}$ and $\mathbf{E}$ ) Iba1+ leukocytes/microglia and GFAP' astrocytes in brainstem regions outside overt lesions in control- and pexidartinib-treated control and Ndufs4(KO) mice (representative images in Figure 2). Data points represent individual animals. $n=4$ for untreated Ndufs 4 (KO), 3 for other groups. ${ }^{*} P<0.05,{ }^{* *} P<0.005$, ${ }^{* * *} P<0.0005$, and ${ }^{* * *} P<0.0001,2$-way ANOVA with Tukey's multiple-testing correction-adjusted $P$ values for pairwise comparisons. (F) Rotarod-induced seizure frequency at $\mathrm{P} 30$ by treatment. ${ }^{*} P<0.016$, Fisher's exact test (Bonferroni-adjusted $P$ value cutoff for significance $=0.0167)$. $n$ indicated by bars. $(\mathbf{G})$ Time to seizure in rotarod assay, P30. All data points shown. ${ }^{*} P<0.05$, log-rank test. $n s$ as in $\mathbf{F}$. $(\mathbf{H})$ Scatter plots of Ndufs4(KO) mouse weight as a function of age and treatment, with local regression (Lowess) curves to display population trends. (I) Cachexia onset (see Figure 1, Methods) in control- and pexidartinib-treated Ndufs4(KO) mice. Colors and ns as in G. ${ }^{*} P<0.0167$, ${ }^{* * *} P<0.00005$, log-rank test (Bonferroni-corrected $P$ value cutoff for significance $=0.0167)$. $(\mathrm{J})$ Blood glucose by age in control- and pexidartinib-treated Ndufs4(KO) animals. Each point represents the median value for 1 animal during the period (data points are biological replicates/individual animals). ${ }^{* * * *} P<0.0001$ by unpaired, unequal variances (Welch's) $t$ test (Bonferroni-corrected $P$ value cutoff for significance $=0.0125$ ). $n$ s as indicated in bars. (K and $\mathbf{L}$ ) Blood lactate in response to a glucose bolus $(2 \mathrm{~g} / \mathrm{kg}$ ) in control and Ndufs4(KO) mice at predisease (P25) and early disease (P45). (K) Time course and (L) total AUC for blood lactate 0-90 minutes. ${ }^{*} P<0.008$, 2-way ANOVA with Tukey's multiple-testing correction-adjusted $P$ values for pairwise comparisons. $n s$ indicated in bars. (M-0) Blood lactate in response to glucose bolus $(2 \mathrm{~g} / \mathrm{kg}$ ) in untreated control and Ndufs4(KO) mice and Ndufs4(KO) mice treated with pexidartinib (300 mg/kg/d in chow), IPI-549 $(100 \mathrm{mg} / \mathrm{kg} / \mathrm{d}$ in chow), or rapamycin (ABI-009 formulation, $8 \mathrm{mg} / \mathrm{kg} / \mathrm{d} \mathrm{IP})$. Time course of blood lactate (M), baseline lactate (N), and total AUC for blood lactate (0) $0-90$ minutes. ${ }^{*} P<0.0167,{ }^{* *} P<0.005$, ${ }^{* * *} P<0.0001$ by unpaired, unequal variances (Welch's) $t$ test (Bonferroni-adjusted significance cutoff $=$ 0.0167). $n$ s indicated in bars ( $\mathbf{N}$ and $\mathbf{~} \mathbf{)}$ ). (P) Change in blood lactate in control and Ndufs $4(\mathrm{KO})$ mice after 30 -minute exposure to $0.4 \%$ isoflurane and impact of treatment with $300 \mathrm{mg} / \mathrm{kg} / \mathrm{d}$ pexidartinib. $n=5$, 7, and $3 .{ }^{* *} P<0.005$ by 1-way ANOVA with Tukey's multiple-testing correction-adjusted $P$ values for pairwise comparisons. (K and $\mathbf{M}$ ) AUC, not individual time points, were compared. (Q) Minimum alveolar anesthetic concentration (MAC) of isoflurane associated with anesthesia in control- and pexidartinib-treated Ndufs4(KO) mice (see Methods). ${ }^{*} P<0.005$ by unpaired, unequal variances (Welch's) $t$ test. $n=$ 6/group. Data represent mean, error bars \pm SEM, unless otherwise stated. 
hepatic damage $(38,39)$, in treated and untreated mice to determine whether hepatotoxicity may contribute to early mortality. Consistent with this possibility, pexidartinib-treated control and $N d u f s 4(\mathrm{KO})$ mice at approximately P150 showed significantly elevated ALT and AST compared with approximately P150 untreated controls [note: $N d u f s 4(\mathrm{KO})$ mice perished prior to this age] (Figure 4, D and E).

Inflammatory chemokines are increased in $N d u f s 4(K O)$ brainstem at ages associated with disease. Finally, a cursory analysis of chemokines in brainstem supports both immune involvement and a post-P37 onset (detailed in Supplemental Figure 9). Among a targeted set of chemokines, 4 showed significantly altered expression in $N d u f s 4(\mathrm{KO})$ mice compared with controls. At P25, before onset of overt disease, only the eosinophil-associated molecule Eotaxin was significantly elevated. At P45 IFN- $\gamma$, IFN- $\gamma$-induced protein 10 (IP-10/CXCL10), and leukemia inhibitory factor were all increased in $N d u f 4(\mathrm{KO})$ mice, while VEGF was reduced. Detailed exploration of immune involvement in LS is beyond the scope of this manuscript, but this analysis identifies putative chemokine mediators that should be of significant interest for follow-up study.

A model for the pathogenesis of Leigh syndrome. Symptoms develop in the Ndufs4(KO) postnatally (see Figure 1), reminiscent of human patients, who are often healthy at birth. Neuron-specific (Nestin-Cre) or glutamatergic neuron-specific (VGlut2-Cre) deletion of $N d u f s 4$ recapitulates disease, including CNS lesions, cachexia, metabolic dysfunction, neuroinflammation, and reduced survival, while $N d u f s 4$ deletion in other neuron types does not $(8,40,41)$. Considering these data, we can assemble a model for the pathogenesis of disease in the $N d u f s 4(\mathrm{KO})$ : mitochondrial dysfunction interacts with development to precipitate cellular sequelae in VGlut $2^{+}$neurons. Glutamatergic neuron dysfunction leads to immune activation and leukocyte recruitment/proliferation with chemokine induction. Leukocyte accumulation is subsequently a causal force in CNS lesions, astrocytosis, CNS dysfunction, and many systemic symptoms (see Figure 4). The final sentence is the key finding of this study.

\section{Discussion}

A causal role for leukocyte proliferation in the pathobiology of LS. Our key findings are that leukocytes are a causal cellular mediator of the pathogenesis of LS and that targeting leukocytes prevents CNS lesions and substantially mitigates disease. Simply put, we have provided evidence that the immune system mechanistically drives pathology in this model of LS, necessitating a reconsideration of current models for the pathobiology of the disease.

Leukocyte depletion not only prevented sequelae associated with overt CNS lesions (e.g., respiratory dysfunction, balance, survival) but also sequelae not attributed to the CNS lesions - hypoglycemia, cachexia, hyperlactemia in response to glucose or anesthesia, seizures, and so on. Whether this indicates these symptoms are downstream of CNS involvement, or peripheral leukocyte activity drives symptoms, remains to be determined.

The rescue of glucose- and isoflurane-induced hyperlactemia was, perhaps, the most unexpected benefit of pexidartinib. This finding indicates that leukocytes mediate increased blood lactate either directly (via leukocyte cellular metabolism) or indirectly, via the consequences of inflammation (e.g., tissue ischemia or metaboregulatory consequences of CNS degeneration). Distinguishing these possibilities will be important in future studies. Additionally, the lactate findings may prove relevant to other forms of GMD involving hyperlactemia.

Evidence for peripheral leukocytes in LS CNS lesions. While microglia are a likely suspect in driving CNS pathologies, we present our findings in terms of leukocytes as a broad category; Iba1, rapamycin, IPI-549, and pexidartinib are not specific to microglia, and our data do not distinguish between microglia and peripheral leukocytes.

LS lesions have been defined by "microgliosis" since the initial description of the disease by Denis Leigh in 1951, but it is critical to note that this designation was based on Nissl staining, which is nonspecific (42). Iba1 staining is, unfortunately, not microglia specific - Iba1 is expressed in all macrophages and has recently been termed a pan-macrophage marker $(43,44)$ - but studies in $N d u f s 4(\mathrm{KO})$ animals have relied on Iba1 staining to identify "microgliosis" without ever noting the lack of microglia specificity (41). Accordingly, use of microgliosis in describing LS lesions is misleading — evidence does support the presence of microglia but does not rule out involvement of nonmicroglial $\mathrm{Iba} 1^{+}$cells.

A detailed investigation of the immune component of LS lesions is a critical next step but is beyond the scope of this manuscript. However, preliminary evidence indicates that peripheral leukocytes contribute significantly to LS lesions: brains of $N d u f s 4(\mathrm{KO})$ mice expressing TMEM119-GFP (a microglia-specific marker, refs. 45, 46) costained for GFP and Iba1 revealed that a substantial portion of cells within lesions 
A

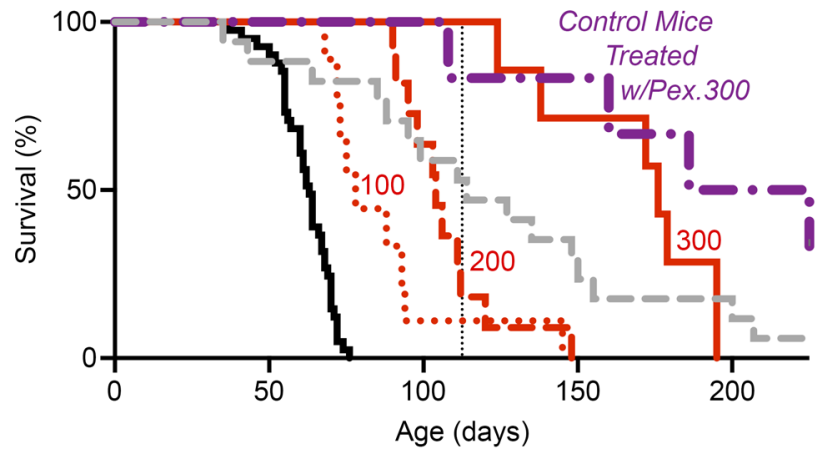

B

\begin{tabular}{|c|c|c|c|c|c|c|}
\hline \multirow{3}{*}{$\begin{array}{l}* * * \\
* * * \\
* * * \\
* * \\
* * * \\
* * * \\
*\end{array}$} & \multirow[b]{2}{*}{ Genotype } & \multirow[b]{2}{*}{ Treatment } & \multirow[b]{2}{*}{ Target } & \multicolumn{3}{|c|}{ Approximate } \\
\hline & & & & $\begin{array}{l}\text { PPM in } \\
\text { Chow }\end{array}$ & $\begin{array}{l}\text { Oral Dose } \\
\text { (mg/kg/day) }\end{array}$ & $\begin{array}{l}\text { Median } \\
\text { Lifespan }\end{array}$ \\
\hline & Ndufs4 (KO) & Control chow & $\mathrm{n} / \mathrm{a}$ & $\mathrm{n} / \mathrm{a}$ & $\mathrm{n} / \mathrm{a}$ & 63 \\
\hline 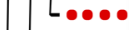 & Ndufs4 (KO) & Pexidartinib & CSF1R & 667 & 100 & 78 \\
\hline$L=$ & Ndufs4 (KO) & Pexidartinib & CSF1R & 1334 & 200 & 104 \\
\hline 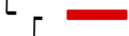 & Ndufs4 (KO) & Pexidartinib & CSF1R & 2001 & 300 & 176 \\
\hline$-\infty$ & Ndufs4 (KO) & $\begin{array}{l}\text { IP Rapamycin } \\
\text { (Science, 2013) }\end{array}$ & mTOR & $n / a$ & 8 mg/kg/day IP & 114 \\
\hline L & - Control & Pexidartinib & CSF1R & 2001 & 300 & 205.5 \\
\hline
\end{tabular}

C

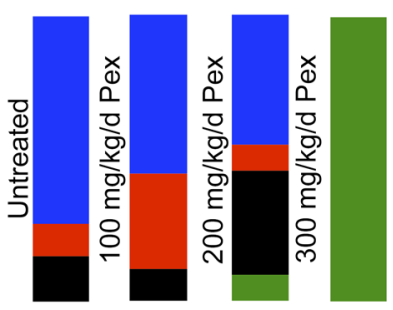

$\square$ Euthanized - weight cutoff

$\square$ Euthanized - moribund

- Severe neurologic symptoms

present, COD unknown

$\square$ Severe neurologic disease

absent, COD unknown

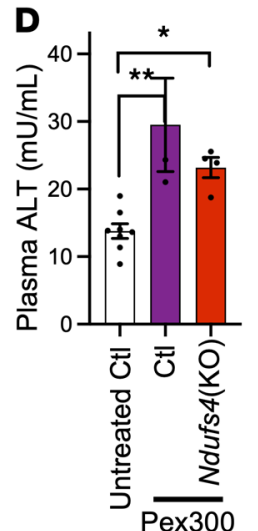

\section{E}

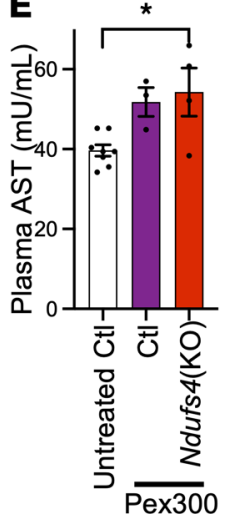

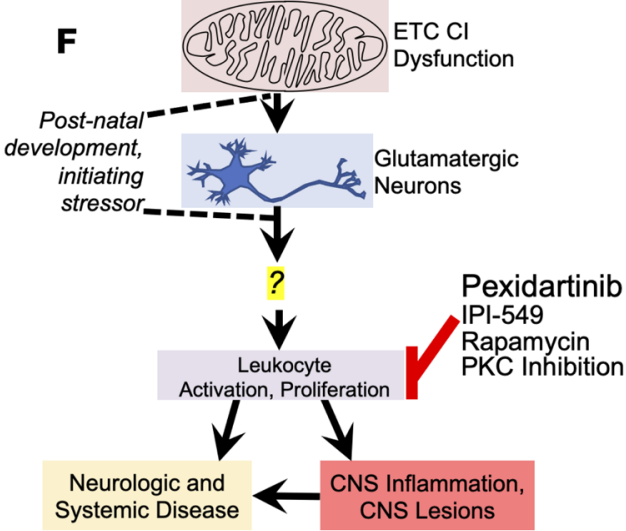

Pexidartinib

PPl-549

Rapamycin

PKC Inhibition

Systemic Disease

CNS Lesions

Figure 4. Pexidartinib dose dependently increases Ndufs4(KO) survival, with survival limited by drug toxicity rather than CNS disease. (A and B) Survival and cause of death in Ndufs 4 (KO) mice treated with increasing doses of pexidartinib. (A) Survival curves. Black line - control-treated Ndufs4(KO). Red dotted, dashed, and solid lines - Ndufs4(KO) mice treated with 100, 200, or $300 \mathrm{mg} / \mathrm{kg} / \mathrm{d}$ pexidartinib, respectively ( $n$ as in Figure 2, G-I). Purple dashed/dotted line - control animals treated with $300 \mathrm{mg} / \mathrm{kg} / \mathrm{d}$ pexidartinib $(n=6)$. Gray dashed line - rapamycin life span (for reference, see Figure 1). (B) Median life spans and dosing data associated with A. ${ }^{* * *} P<0.0001$ by log-rank test (passing Bonferroni's cutoff of $\left.P<0.0167\right)$. (C) Cause of death for Ndufs $4(\mathrm{KO})$ animals in control and pexidartinib treatment groups (all control animals on pexidartinib $300 \mathrm{mg} / \mathrm{kg} / \mathrm{d}$ died of unknown causes with no overt signs of disease/illness). $n$ as in A. (D) Plasma ALT and (E) plasma AST levels (see Supplemental Methods). Data represent mean, error bars \pm SEM. ${ }^{*} P<0.05,{ }^{* *} P<0.005$ by 1-way ANOVA with Tukey's multiple-testing correction-adjusted $P$ values for pairwise comparisons. $n=8$, 3 , and 4 for untreated controls and pexidartinib $300 \mathrm{mg} / \mathrm{kg} / \mathrm{d}$ treated controls and Ndufs $4(\mathrm{KO})$ animals, respectively. (F) A model for the pathogenesis of disease in LS (see Discussion). In this model, CNS lesions and many systemic sequelae are causally downstream of immune involvement. A central role for glutamatergic neurons in initiating disease has previously been identified $(8,40)$, while our findings here reveal a role for leukocytes in the pathogenesis of disease. In the model resulting from these combined data, the mechanisms underlying the benefits of rapamycin and of PKC inhibitors, previously shown to benefit the Ndufs4(KO) mouse, can be accounted for by their convergence with pexidartinib and IPI-549 on leukocyte suppression. Key remaining questions are presented in Discussion.

are Iba1+ but TMEM119- (Supplemental Figure 10 and Supplemental Methods). These data reveal that peripheral immune cells are important contributors to LS lesions. Defining the cellular composition of LS CNS lesions may be critically important to understanding this disease.

A mechanism for $m$ TOR inhibition in LS. Determining the mechanism underlying the benefits of rapamycin in the $N d u f s 4(\mathrm{KO})$ model has been an active area of research since the 2013 study. Processes including metabolism, neurotransmitter abundance, PKC activity, and regulation of transcription have been implicated, but no single downstream pathway has recapitulated the benefits of mTOR suppression (4-6, 8-11, 47-52). Our data support a model whereby these benefits result primarily from immune modulation. Critically, it does not appear any of the prior studies on mTOR in $N d u f s 4(\mathrm{KO})$ animals are incompatible with our findings; some even hinted at immune involvement (for example, the PKC study authors note that PKC inhibitors play a role in mTOR-mediated immune regulation, ref. 11).

It is important to note that mTOR inhibitors have beneficial effects in a variety of GMD models, including cultured cells and invertebrates $(6,48-52)$. mTOR inhibition is pleiotropic, and other mTOR-regulated processes may mediate the benefits of targeting mTOR in other settings. On the other hand, immune activation may play a previously unappreciated role in forms of GMD other than LS, a possibility warranting further attention.

We cannot rule out the possibility that $\mathrm{p} 110 \alpha, \mathrm{p} 110 \beta$, and $\mathrm{p} 110 \delta$ play some minor role in disease. Alternative targeting methods/agents might be more effective. Conversely, the modest benefits observed with the 
$\mathrm{p} 110 \alpha, \mathrm{p} 110 \beta$, and $\mathrm{p} 110 \delta$ inhibitors might simply result from off-target effects on $\mathrm{p} 110 \gamma$. Targeted manipulation of mTOR, p110\%, or CSF1R in leukocytes and/or microglia may provide a more potent benefit with reduced off-target effects, and such experiments might provide further clarity to the pharmacologic results.

Therapeutic implications. Our findings may have significant therapeutic implications. Clinical translation is exceptionally difficult in rare diseases (53-56), compounded in LS by postnatal onset, difficulties in diagnosis, and clinical and genetic heterogeneity (>75 causal genes) (57-59). mTOR inhibition was the first pharmacologic agent shown to attenuate disease in the $N d u f s 4(\mathrm{KO})$ model and benefits at least some patients $(6,7,48)$. However, the translational potential of mTOR inhibitors is limited by incomplete rescue and well-documented off-target effects, among other issues.

We do not suggest that the specific compounds used here will, or should, be trialed in patients. It is the discovery that leukocytes mediate disease which opens the door for an entirely new realm of therapeutic targets. Many well-characterized, clinically approved agents already exist for targeting immune function. We utilized CSF1R inhibition to deplete leukocytes, but this strategy was an experimental tool — untargeted and extreme. Follow-up studies are likely to identify novel agents targeting immune activation in LS with improved potency and reduced off-target effects.

Our findings may apply to other GMDs where inflammation has been observed. Determining the generalizability of our findings to genetically distinct forms of LS and other GMDs will be an important goal of future studies.

New insights, new questions. Our data appear to resolve some mysteries that have persisted in the study of LS. In particular: 1) Why is there a general sparing of some tissues with high energy requirements, as in cardiac-specific $N d u f s 4(\mathrm{KO})$ in the absence of a stressor (60-62)? If disease sequelae are mediated by immune responses, tissue energetics may not play a direct role. 2) Why does LS typically develop postnatally, without symptoms at birth? Viral infection and fever have been reported to often coincide with symptom onset in LS and other forms of acute focal necrotizing encephalopathy in children (63-69), a link attributed by some to "energetic stress" induced by mobilizing an immune response (65). Our data might indicate that immune induction directly contributes to this link, rather than by precipitating an energetic crisis.

While we believe our data represent a significant step forward in our understanding of LS, and perhaps GMD broadly, key questions remain. What precise VGlut $2^{+}$cells initiate inflammation? Do any non-CNS sequelae result from peripheral inflammation? What role, if any, do leukocytes play in other forms of GMD? Is there a link between inflammation and chronic hypoxia, the most potent therapeutic strategy yet identified in the $N d u f s 4(\mathrm{KO})$ model (70)? Finally, how does altered mitochondrial function drive immune activation? One might hypothesize that mitochondrial dysfunction leads to sensing of some mitochondrial component, perhaps mtDNA or misfolded proteins, as "foreign" by the immune machinery. The cyclic GMP-AMP synthase/stimulator of IFN genes pathway, which senses intracellular DNA and induces type $1 \mathrm{IFN}$, provides one candidate for further study but will require identification/isolation of the initiating cell type(s) $(71,72)$. If an immune-activating factor, or factors, can be identified, they may provide additional insight into the curious postnatal onset and unique CNS involvement in LS.

Answers to these questions will shed new light on the interplay between mitochondria, the immune system, and disease. Future studies utilizing targeted genetic manipulation of key immune components will be particularly important in clearly defining the role of the immune system in LS.

\section{Methods}

Animal sources and protocol. $N d u f \mathrm{~S}^{+/-}$mice (originally obtained from the Palmiter laboratory, University of Washington, Seattle, Washington, USA; available through The Jackson Laboratory, strain 027058) were bred to produce $N d u f s 4(\mathrm{KO})\left(N d u f 4^{-1}\right)$ offspring. Mice were weaned at 20-21 days of age. KO mice were housed with control littermates for warmth and stimulation. Mice were weighed and health was assessed a minimum of 3 times per week (daily for i.p.-injected mice; see below). Where longitudinal blood point-ofcare data were collected, this was performed during health checks. Wetted chow in a dish on the bottom of each cage, and in-cage water bottles, were provided to cages housing $N d u f s 4(\mathrm{KO})$ mice following onset of symptoms so that food and water accessibility was never a limiting factor for survival.

Staining in Supplemental Figure 9 used an $N d u f s 4(\mathrm{KO})$ mouse expressing TMEM119-GFP (carrying 1 copy). This reporter line was obtained from The Jackson Laboratory (strain 031823, C57BL/6 strain) and bred into the $N d u f s 4(\mathrm{KO})$ line. 
Mice were euthanized if they reached a $20 \%$ loss in maximum body weight or were immobile or found prostrate or moribund.

As previously reported, $N d u f s 4$ deletion is a recessive defect, and heterozygosity results in no reported phenotypes and no detectable defects in ETC CI activity; controls for this data set included both heterozygous and WT mice.

All experimental mice were fed PicoLab Diet 5058; pharmacologic agents were compounded into this diet (see Supplemental Methods).

Study approval. Animal experiments followed Seattle Children's Research Institute guidelines, and experiments were performed as approved by the IACUC of Seattle Children's Research Institute.

\section{Author contributions}

SCJ conceived the study. SCJ, JC Stokes, RLB, KJ, NAB, KYP, and KAS designed the methodology. SCJ, JC Stokes, RLB, KJ, NB, KYP, KAS, BMJ, JC Snell, and KV investigated. SCJ, RLB, and NAB visualized data. SCJ and MS acquired funding. SCJ performed project administration. SCJ, MMS, and PGM supervised. SCJ wrote the original draft. SCJ, MMS, PGM, and RLB reviewed and edited the draft. For authorship order among co-first authors: order was assigned based on relative contributions and was agreed on by all co-first authors.

\section{Acknowledgments}

We dedicate this study to Alexandra Serex, Steve and Teresa Serex, their family, and all those living with the consequences of mitochondrial disease. They continue to inspire us.

Funding: National Institutes of Health grant NIH/GM R00-126147 (SCJ). National Institutes of Health grant NIH/GM R01-133865 (MS and SCJ). Northwest Mitochondrial Research Guild (SCJ). North American Mitochondrial Disease Consortium (JS and SCJ). National Institutes of Health T32 GM086270 (KS).

Address correspondence to: Simon C. Johnson, Seattle Children's Research Institute, 1900 9th Ave., JMB-925, Seattle, Washington 98101, USA. Phone: 206.660.3172; Email: simoncj@u.washington.edu.

1. Goncalves FG, et al. Primary mitochondrial disorders of the pediatric central nervous system: neuroimaging findings. Radiographics. 2020;40(7):2042-2067.

2. Alston CL, et al. The genetics of mitochondrial disease: dissecting mitochondrial pathology using multi-omic pipelines. $J$ Pathol. 2021;254(4):430-442.

3. Lake NJ, et al. Leigh syndrome: neuropathology and pathogenesis. J Neuropathol Exp Neurol. 2015;74(6):482-492.

4. Johnson SC, et al. Dose-dependent effects of mTOR inhibition on weight and mitochondrial disease in mice. Front Genet. $2015 ; 6: 247$

5. Johnson SC, et al. mTOR inhibition alleviates mitochondrial disease in a mouse model of Leigh syndrome. Science. 2013;342(6165):1524-1528.

6. Johnson SC, et al. mTOR inhibitors may benefit kidney transplant recipients with mitochondrial diseases. Kidney Int. 2019;95(2):455-466

7. Sage-Schwaede A, et al. Exploring mTOR inhibition as treatment for mitochondrial disease. Ann Clin Transl Neurol. 2019;6(9):1877-1881.

8. Johnson SC, et al. Regional metabolic signatures in the Ndufs4(KO) mouse brain implicate defective glutamate/ $\alpha$-ketoglutarate metabolism in mitochondrial disease. Mol Genet Metab. 2020;130(2):118-132.

9. Ito TK, et al. Hepatic S6K1 partially regulates lifespan of mice with mitochondrial complex I deficiency. Front Genet. 2017;8:221.

10. Lee CF, et al. Targeting NAD ${ }^{+}$metabolism as interventions for mitochondrial disease. Sci Rep. 2019;9(1):3073.

11. Martin-Perez M, et al. PKC downregulation upon rapamycin treatment attenuates mitochondrial disease. Nat Metab. 2020;2(12):1472-1481.

12. Saudemont A, et al. p110gamma and p110delta isoforms of phosphoinositide 3-kinase differentially regulate natural killer cell migration in health and disease. Proc Natl Acad Sci U S A. 2009;106(14):5795-5800.

13. Fritsch $C$, et al. Characterization of the novel and specific PI3K $\alpha$ inhibitor NVP-BYL719 and development of the patient stratification strategy for clinical trials. Mol Cancer Ther. 2014;13(5):1117-1129.

14. Leroy C, et al. Activation of IGF1R/p110 /AKT/mTOR confers resistance to $\alpha$-specific PI3K inhibition. Breast Cancer Res. 2016;18(1):41.

15. Pridham KJ, et al. PIK3CB/p110 $\beta$ is a selective survival factor for glioblastoma. Neuro Oncol. 2018;20(4):494-505.

16. Davis RJ, et al. Anti-PD-L1 efficacy can be enhanced by inhibition of myeloid-derived suppressor cells with a selective inhibitor of $\mathrm{PI} 3 \mathrm{~K} \delta / \gamma$. Cancer Res. 2017;77(10):2607-2619.

17. Bornstein R, et al. Differential effects of mTOR inhibition and dietary ketosis in a mouse model of subacute necrotizing 
encephalomyelopathy. Neurobiol Dis. 2021;163:105594.

18. Zhao JJ, et al. The p110alpha isoform of PI3K is essential for proper growth factor signaling and oncogenic transformation. Proc Natl Acad Sci U S A. 2006;103(44):16296-16300.

19. Burger MT, et al. Identification of NVP-BKM120 as a potent, selective, orally bioavailable class I PI3 kinase inhibitor for treating cancer. ACS Med Chem Lett. 2011;2(10):774-779.

20. Schubert Baldo M, Vilarinho L. Molecular basis of Leigh syndrome: a current look. Orphanet J Rare Dis. 2020;15(1):31.

21. Gonzalez-Angulo AM, et al. Weekly nab-Rapamycin in patients with advanced nonhematologic malignancies: final results of a phase I trial. Clin Cancer Res. 2013;19(19):5474-5484.

22. Denny WA, Flanagan JU. Small-molecule CSF1R kinase inhibitors; review of patents 2015-present. Expert Opin Ther Pat. 2021;31(2):107-117.

23. Quintana A, et al. Fatal breathing dysfunction in a mouse model of Leigh syndrome. J Clin Invest. 2012;122(7):2359-2368

24. Ramirez SC, et al. Perinatal breathing patterns and survival in mice born prematurely and at term. Front Physiol. 2019;10:1113.

25. Lee HF, et al. Epileptic seizures in infants and children with mitochondrial diseases. Pediatr Neurol. 2011;45(3):169-174.

26. Cameron JM, et al. Exome sequencing identifies complex I NDUFV2 mutations as a novel cause of Leigh syndrome. Eur J Paediatr Neurol. 2015;19(5):525-532.

27. Fukumura S, et al. Compound heterozygous GFM2 mutations with Leigh syndrome complicated by arthrogryposis multiplex congenita. J Hum Genet. 2015;60(9):509-513.

28. Yokoyama J, et al. [A case of rhabdomyolysis after status epilepticus without stroke-like episodes in mitochondrial myopathy, encephalopathy, lactic acidosis, and stroke-like episodes]. Rinsho Shinkeigaku. 2016;56(3):204-207.

29. Yamada K, et al. Diagnostic accuracy of blood and CSF lactate in identifying children with mitochondrial diseases affecting the central nervous system. Brain Dev. 2012;34(2):92-97.

30. Chi CS, et al. Lactate peak on brain MRS in children with syndromic mitochondrial diseases. J Chin Med Assoc. 2011;74(7):305-309.

31. Takahashi Y, et al. Detection of increased intracerebral lactate in a mouse model of Leigh syndrome using proton MR spectroscopy. Magn Reson Imaging. 2019;58:38-43.

32. Nafisinia M, et al. Whole exome sequencing identifies the genetic basis of late-onset Leigh syndrome in a patient with MRI but little biochemical evidence of a mitochondrial disorder. JIMD Rep. 2017;32:117-124.

33. Kramer PA, et al. A review of the mitochondrial and glycolytic metabolism in human platelets and leukocytes: implications for their use as bioenergetic biomarkers. Redox Biol. 2014;2:206-210.

34. Hsieh VC, et al. Anesthetic hypersensitivity in a case-controlled series of patients with mitochondrial disease. Anesth Analg 2021;133(4):924-932.

35. Kayser EB, et al. GAS-1: a mitochondrial protein controls sensitivity to volatile anesthetics in the nematode Caenorhabditis elegans. Anesthesiology. 1999;90(2):545-554.

36. Sonner JM, et al. Naturally occurring variability in anesthetic potency among inbred mouse strains. Anesth Analg. 2000;91(3):720-726

37. Lewis $\mathrm{JH}$, et al. Pexidartinib long-term hepatic safety profile in patients with tenosynovial giant cell tumors. Oncologist. 2021;26(5):e863-e873.

38. Ikeda H, et al. PI3K/p110 is a novel therapeutic target in multiple myeloma. Blood. 2010;116(9):1460-1468.

39. Carnevalli LS, et al. PI3K $\alpha / \delta$ inhibition promotes anti-tumor immunity through direct enhancement of effector CD $8^{+} \mathrm{T}$-cell activity. J Immunother Cancer. 2018;6(1):158.

40. Bolea I, et al. Defined neuronal populations drive fatal phenotype in a mouse model of Leigh syndrome. Elife. 2019;8:e47163.

41. Quintana A, et al. Complex I deficiency due to loss of Ndufs4 in the brain results in progressive encephalopathy resembling Leigh syndrome. Proc Natl Acad Sci U S A. 2010;107(24):10996-11001.

42. Leigh D. Subacute necrotizing encephalomyelopathy in an infant. J Neurol Neurosurg Psychiatry. 1951;14(3):216-221.

43. Kohler C. Allograft inflammatory factor-1/Ionized calcium-binding adapter molecule 1 is specifically expressed by most subpopulations of macrophages and spermatids in testis. Cell Tissue Res. 2007;330(2):291-302.

44. Pierezan F, et al. Immunohistochemical expression of ionized calcium binding adapter molecule 1 in cutaneous histiocytic proliferative, neoplastic and inflammatory disorders of dogs and cats. J Comp Pathol. 2014;151(4):347-351.

45. Kaiser T, Feng G. Tmem119-EGFP and Tmem119-CreERT2 transgenic mice for labeling and manipulating microglia. eNeuro. 2019;6(4):ENEURO.0448-18.2019.

46. van Wageningen TA, et al. Regulation of microglial TMEM119 and P2RY12 immunoreactivity in multiple sclerosis white and grey matter lesions is dependent on their inflammatory environment. Acta Neuropathol Commun. 2019;7(1):206.

47. Johnson SC. Translational medicine. A target for pharmacological intervention in an untreatable human disease. Science. 2014;346(6214):1192.

48. Siegmund SE, et al. Low-dose rapamycin extends lifespan in a mouse model of mtDNA depletion syndrome. Hum Mol Genet. 2017;26(23):4588-4605.

49. Civiletto G, et al. Rapamycin rescues mitochondrial myopathy via coordinated activation of autophagy and lysosomal biogenesis EMBO Mol Med. 2018;10(11):e8799.

50. Wischhof L, et al. A disease-associated Aifm1 variant induces severe myopathy in knockin mice. Mol Metab. 2018;13:10-23.

51. Wang A, et al. Rapamycin enhances survival in a Drosophila model of mitochondrial disease. Oncotarget. 2016;7(49):80131-80139.

52. Fan F, et al. Rapamycin as a potential treatment for succinate dehydrogenase deficiency. Heliyon. 2019;5(2):e01217.

53. Dawkins HJS, et al. Progress in rare diseases research 2010-2016: an IRDiRC perspective. Clin Transl Sci. 2018;11(1):11-20.

54. Gopal-Srivastava R, Kaufmann P. Facilitating clinical studies in rare diseases. Adv Exp Med Biol. 2017;1031:125-140.

55. Kaufmann P, et al. From scientific discovery to treatments for rare diseases - the view from the National Center for Advancing Translational Sciences — office of rare diseases research. Orphanet J Rare Dis. 2018;13(1):196.

56. Rath A, et al. A systematic literature review of evidence-based clinical practice for rare diseases: what are the perceived and real barriers for improving the evidence and how can they be overcome? Trials. 2017;18(1):556.

57. Chang X, et al. A meta-analysis and systematic review of Leigh syndrome: clinical manifestations, respiratory chain enzyme complex deficiency, and gene mutations. Medicine (Baltimore). 2020;99(5):e18634 
58. Pitceathly RDS, et al. Moving towards clinical trials for mitochondrial diseases. J Inherit Metab Dis. 2021;44(1):22-41.

59. Lee JS, et al. Genetic heterogeneity in Leigh syndrome: highlighting treatable and novel genetic causes. Clin Genet. 2020;97(4):586-594

60. Karamanlidis G, et al. Mitochondrial complex I deficiency increases protein acetylation and accelerates heart failure. Cell Metab. 2013;18(2):239-250

61. Zhang H, et al. Heart specific knockout of Ndufs4 ameliorates ischemia reperfusion injury. J Mol Cell Cardiol. 2018;123:38-45

62. Chiao YA, et al. NAD ${ }^{+}$Redox imbalance in the heart exacerbates diabetic cardiomyopathy. Circ Heart Fail. 2021;14(8):e008170.

63. Edmonds JL, et al. The otolaryngological manifestations of mitochondrial disease and the risk of neurodegeneration with infection. Arch Otolaryngol Head Neck Surg. 2002;128(4):355-362.

64. Lee YJ, et al. Acute necrotizing encephalopathy in children: a long way to go. J Korean Med Sci. 2019;34(19):e143.

65. Niyazov DM, et al. Primary mitochondrial disease and secondary mitochondrial dysfunction: importance of distinction for diagnosis and treatment. Mol Syndromol. 2016;7(3):122-137.

66. Porta F, et al. SLC25A19 deficiency and bilateral striatal necrosis with polyneuropathy: a new case and review of the literature. J Pediatr Endocrinol Metab. 2021;34(2):261-266.

67. Wang HS, Huang SC. Acute necrotizing encephalopathy of childhood. Chang Gung Med J. 2001;24(1):1-10.

68. Wei Y, Wang L. Adult-onset Leigh syndrome with central fever and peripheral neuropathy due to mitochondrial $9176 \mathrm{~T}>\mathrm{C}$ mutation. Neurol Sci. 2018;39(12):2225-2228.

69. Wu TS, et al. Leigh disease (subacute necrotizing encephalomyelopathy): report of one case. Zhonghua Min Guo Xiao Er Ke Yi Xue Hui Za Zhi. 1993;34(4):301-307.

70. Jain IH, et al. Hypoxia as a therapy for mitochondrial disease. Science. 2016;352(6281):54-61.

71. Couillin I, Riteau N. STING signaling and sterile inflammation. Front Immunol. 2021;12:753789.

72. Zhu Y, et al. STING: a master regulator in the cancer-immunity cycle. Mol Cancer. 2019;18(1):152. 